$\Rightarrow$ GLIA

\section{Remodelling the matrix}

Microglia have been implicated in the remodelling of synaptic connections, microglia from II33 cKO mice showed less engulfed aggrecan than controls

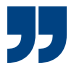

revealed a cluster of neurons in the DG that exhibited enriched expression of genes involved in synapse formation and ECM with neurons shown to be enriched in Il33 expression.

Among mature DG granule cells from Il33 $3^{\text {Cherry/t }}$ mice, those with high mCherry levels, indicative of high IL-33 expression, exhibited remodelling. This cluster overlapped higher levels of spine density and spine head filopodia, a marker of spine plasticity, than those with lower mCherry levels. Neurons with higher mCherry levels were also more likely to show expression of FOS, a marker of neural activity, than other neurons when $I l 33^{\text {mCherry/+ }}$ mice were placed in a novel environment for an hour. These data suggest that IL-33 expression is a feature of neurons with a greater probability of undergoing synaptic remodelling.

Quantitative PCR and in situ hybridization showed that microglia were the main type of hippocampal cell that expressed IL-1 receptor like-1 (IL1RL1), the IL-33 receptor. Mice in which Il33 was conditionally knocked out in neurons (Il33 cKO mice) or Il1rl1 was conditionally knocked out in microglia (Il1rl1 i-cKO mice) showed reduced spine density in the DG and CA1. Expression of a modified form of IL-33 in DG neurons, which allowed constitutive release of the cytokine, was sufficient to increase spine number, but this effect was not observed in Il1rl1 i-cKO mice. These data indicate that spine formation and plasticity is promoted by IL-33-mediated neuron-microglial cell interactions.

Environmental enrichment increases neurogenesis and the integration of newborn neurons into circuits. Il33 cKO mice had fewer newborn neurons than controls after a 5-week exposure to an enriched environment, suggesting IL-33 is required for an experience-dependent rise in newborn neurons.

The authors next examined whether IL-33 has a role in hippocampus-dependent memory. Il33 cKO mice showed good memory recall and context discrimination shortly after training in a contextual fear discrimination task, but they developed a deficit in context discrimination 2-4 weeks later. This suggests that IL-33-mediated neuron-microglial cell interactions are important for memory consolidation.

How does the IL-33 signal affect microglia? Other studies have showed that IL-33 promotes microglial phagocytosis. Here, transcriptional profiling revealed that IL-33-exposed hippocampal microglia were strongly activated and showed upregulation of microglial genes associated with ECM regulation.

Strikingly, high-resolution imaging and $3 \mathrm{D}$ reconstruction revealed immunostaining for aggrecan - an ECM protein — in some lysosomes in microglia. Moreover, microglia from $I l 33$ cKO mice showed less engulfed aggrecan than controls. In line with these findings, Il33 cKO mice showed a higher density of aggrecan and brevican, another ECM protein, perisynaptically in the DG molecular layer than did control mice. Together, these data suggest that neuronal IL-33 promotes microglial phagocytosis, restricting the amount of ECM near synapses.

This study shows that, in the hippocampus of adult mice, neuronally expressed IL-33 promotes microglial cell-mediated ECM remodelling, in turn promoting synaptic plasticity and the consolidation of memories.

Darran Yates

ORIGINAL ARTICLE Nguyen, P. T. et al. Microglial remodelling of the extracellular matrix promotes synapse plasticity. Cell https://doi.org/10.1016/ j.cell.2020.05.050 (2020) 